ACCEPTED for PUblicAtion in ApJ

Preprint typeset using LATEX style emulateapj v. 12/16/11

\title{
A NEW INTERPRETATION OF THE PERIOD-LUMINOSITY SEQUENCES OF LONG-PERIOD VARIABLES
}

\author{
Michele Trabucchi \\ Dipartimento di Fisica e Astronomia Galileo Galilei Università di Padova, Vicolo dell'Osservatorio 3, I-35122 Padova, Italy \\ Peter R. WOOD \\ Research School of Astronomy and Astrophysics, Australian National University, Canberra, ACT2611, Australia \\ Josefina Montalbán \\ Dipartimento di Fisica e Astronomia Galileo Galilei Università di Padova, Vicolo dell'Osservatorio 3, I-35122 Padova, Italy \\ PaOla Marigo \\ Dipartimento di Fisica e Astronomia Galileo Galilei Università di Padova, Vicolo dell'Osservatorio 3, I-35122 Padova, Italy \\ Giada Pastorelli \\ Dipartimento di Fisica e Astronomia Galileo Galilei Università di Padova, Vicolo dell'Osservatorio 3, I-35122 Padova, Italy \\ LÉO GIRARDi \\ Astronomical Observatory of Padova - INAF, Vicolo dell'Osservatorio 3, I-35122 Padova, Italy \\ Accepted for publication in ApJ
}

\begin{abstract}
Period-luminosity (PL) sequences of long period variables (LPVs) are commonly interpreted as different pulsation modes, but there is disagreement on the modal assignment. Here, we re-examine the observed PL sequences in the Large Magellanic Cloud, including the sequence of long secondary periods (LSPs), and their associated pulsation modes. Firstly, we theoretically model the sequences using linear, radial, non-adiabatic pulsation models and a population synthesis model of the LMC red giants. Then, we use a semi-empirical approach to assign modes to the pulsation sequences by exploiting observed multi-mode pulsators. As a result of the combined approaches, we consistently find that sequences $\mathrm{B}$ and $\mathrm{C}^{\prime}$ both correspond to first overtone pulsation, although there are some fundamental mode pulsators at low luminosities on both sequences. The masses of these fundamental mode pulsators are larger at a given luminosity than the mass of the first overtone pulsators. These two sequences $\mathrm{B}$ and $\mathrm{C}^{\prime}$ are separated by a small period interval in which large amplitude pulsation in a long secondary period (sequence D variability) occurs, meaning that the first overtone pulsation is not seen as the primary mode of pulsation. Observationally, this leads to the splitting of the first overtone pulsation sequence into the two observed sequences B and $\mathrm{C}^{\prime}$. Our two independent examinations also show that sequences $\mathrm{A}^{\prime}, \mathrm{A}$ and $\mathrm{C}$ correspond to third overtone, second overtone and fundamental mode pulsation, respectively.
\end{abstract}

Keywords: stars: AGB and post-AGB - stars: oscillations - stars: variables: general - Magellanic Clouds

\section{INTRODUCTION}

Luminous red giants are known to be variable, and their periods of observed variability lie on well defined sequences in period-luminosity (PL) diagrams. Wood et al. (1999) and Wood (2000) identified five sequences (labelled A, B, C, D and E) in the PL diagram of the Large Magellanic Cloud (LMC) using observations from the MACHO project.

Ita et al. (2004) found that sequence B is in fact two sequences, B and $\mathrm{C}^{\prime}$, while Soszynski et al. (2004) showed that an additional sequence exists on the short-period side of sequence A. They labelled that sequence $\mathrm{a}_{4}$, although we use here the alternative designation $\mathrm{A}^{\prime}$, as

michele.trabucchi@phd.unipd.it in Wood (2015) and references therein. Moreover, sequences $\mathrm{A}$ and $\mathrm{B}$, and possibly $\mathrm{A}^{\prime}$, each consist of three or more closely spaced sequences (Soszynski et al. 2004, 2007).

Here, we are interested in sequences $\mathrm{A}^{\prime}, \mathrm{A}, \mathrm{B}, \mathrm{C}^{\prime}$ and $\mathrm{C}$, which are attributed to pulsating stars. All these long period variables (LPVs) can be either on their red giant branch (RGB) or asymptotic giant branch (AGB) phases, with just a slight offset between the respective period distributions (Ita et al. 2002; Kiss \& Bedding 2003). Sequence D consists of long secondary periods (LSPs), while sequence $\mathrm{E}$ is due to binary stars. We do not discuss sequence E further in this paper.

Existing interpretations of the observed sequences $\mathrm{A}^{\prime}$, $\mathrm{A}, \mathrm{B}, \mathrm{C}^{\prime}$ and $\mathrm{C}$ all assume that these sequences corre- 
spond to distinct and adjacent radial orders of pulsation. There are two incompatible interpretations in the literature. Wood (2015) assumed that sequence C, containing the Mira variables, corresponded to the radial fundamental mode so that sequences $\mathrm{C}^{\prime}, \mathrm{B}, \mathrm{A}$ and $\mathrm{A}^{\prime}$ corresponded to the radial 1st, $2 \mathrm{nd}$, 3rd and 4 th overtones, respectively. On the other hand, Mosser et al. (2013) and Soszynski et al. (2007) matched sequences B and A to the 1st and 2 nd radial overtones, meaning that sequence $\mathrm{C}^{\prime}$ is the fundamental mode and that sequence $\mathrm{C}$ has no explanation. Note that there is a one sequence offset between the above two sets of mode assignments.

A solution to the above mode assignment incompatibility is to relax the requirement that the adjacent observed sequences correspond to adjacent radial modes of pulsation, where we identify radial order $n=0$ with the fundamental mode, radial order $n=1$ with the 1 st overtone (1O) mode, and so on.

Here, we explore the possibility that sequence B corresponds to $1 \mathrm{O}$ mode pulsation as suggested by Mosser et al. (2013), sequence C corresponds to radial fundamental mode pulsation as required by Wood (2015), but that sequence $\mathrm{C}^{\prime}$ is associated with $1 \mathrm{O}$ or fundamental radial mode, or both these modes. To do this, we firstly construct theoretical pulsation sequences and compare them with observations, following which we take an independent semi-empirical approach and examine observations of stars exhibiting multiple periods.

\section{PERIOD-LUMINOSITY RELATIONS IN A POPULATION} SYNTHESIS MODEL

\subsection{Methods}

We used the code TRILEGAL (Girardi et al. 2005) to compute a synthetic stellar population representative of the LMC, assuming a constant star formation rate from ages $\sim 5 \mathrm{Myr}$ to $\sim 10 \mathrm{Gyr}$ and the age-metallicity relation by Pagel \& Tautvaišienè (1999). Such a simplifying assumption is made to eliminate discontinuities that could be caused by periods of reduced or null star formation. The technical details of such simulations are discussed elsewhere (Pastorelli et al., in prep.). Here, it suffices to recall that the simulations include single stars in all relevant evolutionary phases, as extracted from the PARSEC (Bressan et al. 2012) and COLIBRI (Marigo et al. 2013) grids of stellar evolutionary tracks. As to the AGB phase, stellar models account for the formation of carbon stars due to the third dredge-up, as well as for the occurrence of hot-bottom burning (Marigo et al. 2017). The simulation output includes many intrinsic stellar quantities such as luminosity, radius, core mass, surface abundances, and evolutionary stage, as well as the apparent photometry.

We then used the radial, non-adiabatic pulsation models described in Wood \& Olivier (2014) and Trabucchi et al., in prep., to assign periods and growth rates for five radial modes to each simulated RGB or AGB star. Models include detailed atomic and molecular opacities (Marigo \& Aringer 2009) for a realistic description of carbon stars. The growth rates provide an indication of the stability of pulsation modes: a mode having a negative growth rate should be stable, while a positive growth rate means a mode is excited. In a given stellar model, we call the dominant mode the one with the largest growth rate, and it is also expected to have the largest amplitude.

\subsection{Comparison with observations}

We compare the results with observations using data from the OGLE III Catalogue of LPVs in the LMC (Soszyński et al. 2009) combined with near-infrared photometry from the Two Micron All Sky Survey (2MASS) Point Source Catalogue (Skrutskie et al. 2006). The comparison is made in the $\log (P)-W_{\mathrm{JK}}$ plane, where the Weisenheit index defined as

$$
W_{\mathrm{JK}}=K_{\mathrm{s}}-0.686\left(J-K_{\mathrm{s}}\right)
$$

is a reddening-free measure of the luminosity.

We use periods derived from radial, non-adiabatic models, and compare the dominant mode (the mode with the highest growth rate) in the models to the observed primary modes in the OGLE data. The latter are the ones associated with the highest peak in the power spectrum, and have generally the largest amplitude. Theoretical modes having a positive but small growth rate are not expected to be observed, therefore only dominant modes with a growth rate larger than 0.04 are shown, where the growth rate is the fractional growth in amplitude per period as defined by Wood \& Olivier (2014). The comparison is shown in Fig. 1.

We find the models to be in reasonably good agreement with observations. The observed sequences can be explained with exactly four radial oscillation modes, from the fundamental to the 30 mode. The radial 40 mode, also computed for the simulation, is never predicted to be dominant, and is not required to reproduce the observations.

Sequences $\mathrm{A}^{\prime}$ and $\mathrm{A}$ are fairly well reproduced by radial $3 \mathrm{O}$ and $2 \mathrm{O}$ mode periods, respectively, although in both cases the theoretical sequences are slightly offset towards short periods with respect to observed ones.

When the radial 10 mode is dominant, it has periods extending across both sequences $\mathrm{B}$ and $\mathrm{C}^{\prime}$. However, there is no theoretical counterpart in the models for the gap between the two sequences (this will be discussed in Section 3.3).

Dominant fundamental periods are distributed mainly to the right of sequence $\mathrm{C}$, and are not as a good match to the observations as are the overtones. However, according to the models, the fundamental mode is the only mode possibly responsible for sequence $\mathrm{C}$, as no other mode can reach such long periods. The reasons for the poor agreement between the positions of sequence $\mathrm{C}$ and the positions of predicted fundamental mode pulsators are explored further in Section 2.3.

\subsection{Amplitudes and growth rates}

Here, we examine observed amplitudes to interpret LPVs in terms of different pulsation modes. In panel (a) of Fig. 2, the distribution of observed amplitudes ${ }^{1}$ is shown against the variable $\log \left(P_{W_{\mathrm{JK}}=12}\right)$ defined by

$$
\log \left(P_{W_{\mathrm{JK}}=12}\right)=\log (P)+\frac{W_{\mathrm{JK}}-12}{4.444}
$$

1 Amplitude data in the OGLE3 catalogue comes rounded to the third decimal digit. As a consequence, visual inspection of low-amplitude distributions in log-scale is virtually impossible. For this reason, we added random errors with a standard deviation of $7.5 \cdot 10^{-4}$ mag to amplitude data, making their distribution visually smooth in Fig. 2. 


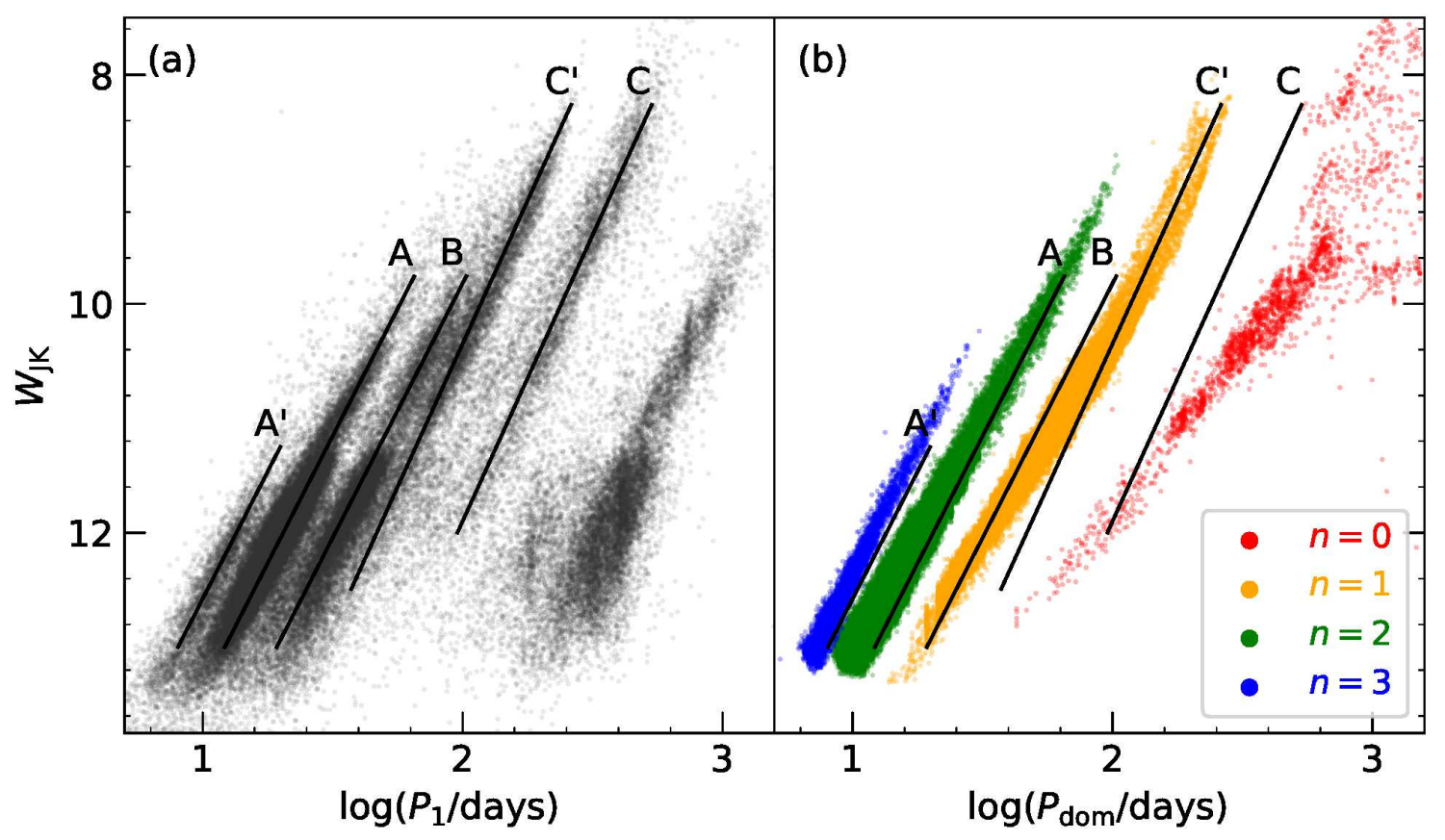

Figure 1. Observed primary periods (panel (a)) are compared to theoretically dominant periods obtained from radial, linear, nonadiabatic models (panel (b)). Different colours represent periods of different modes: red for fundamental mode, orange for 1O, green for $2 \mathrm{O}$ mode, blue for $3 \mathrm{O}$ mode. Solid lines provide a visual aid to identify the approximate location and slope of observed sequences.

which represents $\log (P)$ projected along a line parallel to the observed sequences until it meets the level $W_{\mathrm{JK}}=12$. This way, periods on a given sequence are bounded to the same horizontal range (see also Wood 2015).

Similarly to what happens in the PL diagram, where observations lie on well defined sequences, in Fig. 2 observed modes are distributed into four distinct groups, that are actually period-amplitude-luminosity (PAL) relations (luminosity plays a role through the dependence of $\log \left(P_{W_{\mathrm{JK}}=12}\right)$ upon $W_{\mathrm{JK}}$ in Eq. 2$)$. At short periods $\left(\log \left(P_{W_{\mathrm{JK}}=12}\right) \lesssim 1.2\right)$, the cluster of observed modes of small amplitude is the counterpart of sequence $\mathrm{A}^{\prime}$, while the one with $1.2 \lesssim \log \left(P_{W_{\mathrm{JK}}=12}\right) \lesssim 1.4$, and extending up to larger amplitudes, corresponds to sequence A. Towards long periods, clusters become elongated and slanted. The one starting at $\log \left(P_{W_{\mathrm{JK}}=12}\right) \simeq 1.4$ with small amplitude, corresponding to sequence B, culminates at larger amplitude at $\log \left(P_{W_{\mathrm{JK}}=12}\right) \simeq 1.6$, where it is the counterpart of the bright end of sequence $\mathrm{C}^{\prime}$. Finally, the faint end of sequence $\mathrm{C}^{\prime}$ corresponds to the clump at small amplitude at $\log \left(P_{W_{\mathrm{JK}}=12}\right) \simeq 1.6$, which extends to larger amplitudes at $\log \left(P_{W_{\mathrm{JK}}=12}\right) \simeq 1.9$, corresponding to sequence $\mathrm{C}$. It then shows a tendency to bend back to the short period side of sequence $\mathrm{C}$ at the largest amplitudes, corresponding to Miras, whose amplitude is limited by nonlinear effects.

In the previous subsection, we showed that the region occupied by the sequences in the PL diagram is also reproduced by exactly four radial modes. This suggest that the four PAL relations can be identified with radial modes from the fundamental to the 30 . To support this hypothesis, we use theoretical growth rates as a proxy for mode amplitude in order to make a comparison with the observed distribution of amplitudes, as shown in panel (b) of Fig. 2.

The distribution of growth rates there exhibits a char- acteristic behaviour typical of the evolution of pulsational stability in luminous red giants (this will be discussed in section 3.2, see also Fig. 5). At short periods, the $3 \mathrm{O}$ mode is dominant (has the largest growth rate) and its period falls in the vicinity of sequence $\mathrm{A}^{\prime}$. With further evolution, its growth rate drops, forming the vertical strip at $\log \left(P_{W_{\mathrm{JK}}=12}\right) \simeq 1$. . The $2 \mathrm{O}$ mode shows the same behaviour with changing $\log \left(P_{W_{\mathrm{JK}}=12}\right)$. For the 10 mode, the growth rate rises with $\log \left(P_{W_{\mathrm{JK}}}=12\right)$ as for the $2 \mathrm{O}$ and $3 \mathrm{O}$ modes but the vertical strip corresponding to the growth rates dropping to negative values is absent. The fundamental mode shows a different pattern, being stable or weakly unstable most of the time, and becoming suddenly very unstable (very large growth rates, like those at $\left.\log \left(P_{W_{\mathrm{JK}}=12}\right) \gtrsim 2\right)$.

In Fig. 2, the observed amplitudes seen in panel (a) and the predicted amplitudes seen in panel (b) describe an entirely similar pattern as long as only overtone modes are considered. We interpret this agreement as a validation of our hypothesis to assign a different radial order of oscillation to each PAL relation. It also supports the idea that sequences $\mathrm{B}$ and $\mathrm{C}^{\prime}$ are both due to $1 \mathrm{O}$ mode pulsation. The remaining PAL relation has periods longer than the 10 mode and is related to sequence $\mathrm{C}$, which is almost certainly due to fundamental mode pulsation (Wood 2015). Thus, we identify it with the fundamental mode in spite of the poor agreement of models with observations.

Such a poor agreement is a consequence of the fact that the fundamental mode is generally well reproduced in terms of periods, but not of driving. We further explore this aspect in Section 3.2.

To show that the issue is limited to the modelling of driving, and to validate our identification of PAL relations with pulsation modes, in Fig. 3 we examine the distribution of all theoretical fundamental periods, regard- 


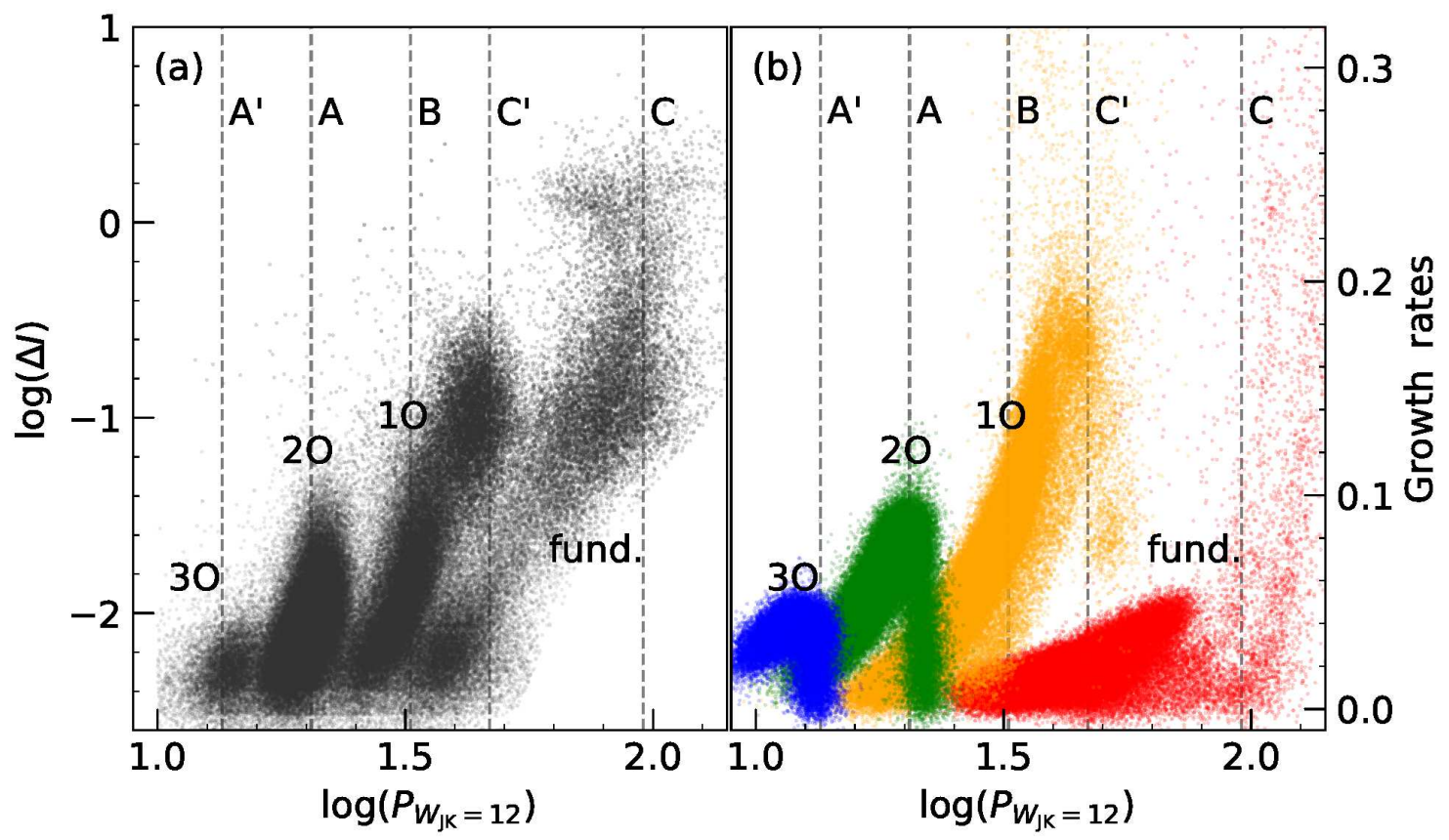

Figure 2. Panel (a): logarithm of observed $I$ band amplitudes, for all three periods of stars in the OGLE3 catalogue, as a function of $\log \left(P_{W_{\mathrm{JK}}=12}\right)$. The distinct PAL relations are labelled with the corresponding mode according to the interpretation provided here (see text). Periods on sequence $\mathrm{D}$ and longer than sequence $\mathrm{C}$ have been ignored, as they are not related to the normal modes of pulsation (see Section 3.1). Panel (b): theoretical growth rates for all excited modes up to the 3O, labelled and colour coded according to their radial order as in Fig. 1. Vertical dashed lines in the background of both panels approximately identify the centre of the PL sequences.

less of their growth rates. We compare this distribution with the PAL relation of longest period (the one labelled "fund."), including primary, secondary and tertiary periods rather than only the primary periods. Fundamental mode periods, both in the observations and in the models, cover not only the region of sequence $\mathrm{C}$, but also a wide area including the the faint part of sequences $B$ and $\mathrm{C}^{\prime}$. The agreement found is a confirmation that fundamental mode periods are generally correct, although slightly overestimated.

\section{SEMI-EMPIRICAL ANALYSIS OF THE PULSATION SEQUENCES OF LPVS}

\subsection{The pulsation modes associated with the observed PL sequences}

In this section, we examine the periods in the many multimode LPVs in the LMC. In Fig. 4 we show six $\log (P)-W_{\mathrm{JK}}$ diagrams for LPVs in the LMC. Black points in the background are observed primary periods, and form the approximately parallel $\mathrm{PL}$ sequences $\mathrm{A}^{\prime}, \mathrm{A}$, $\mathrm{B}, \mathrm{C}^{\prime}$ and $\mathrm{C}$. The sequence identifications are shown on panel (a) of Fig. 4.

Panel (a) of Fig. 4 shows the secondary and tertiary periods (green points) associated with stars whose primary periods (orange points) lie is a channel down the centre of sequence A. There are clearly three additional modes associated with those stars ${ }^{2}$. The most important point to note about panel (a) is that there are only two modes (green strips) with periods longer than the mode asso-

2 We ignore the broad area of green points on the long period side of sequence $\mathrm{C}$ which are a mixture of periods related to the presence of LSPs on sequence D and many other periods caused by the annual observing cycle for the OGLE data and possibly by convection-related variability. ciated with sequence A (sampled by the orange strip). Assuming the longest period mode corresponds to fundamental mode pulsation, then sequence A must correspond to second overtone (2O) pulsation. There is one green strip in panel (a) with a period shorter than that of sequence $\mathrm{A}$ and this must correspond to the $3 \mathrm{O}$ mode of pulsation. Sequence $\mathrm{A}^{\prime}$, which is on the short period side of sequence $\mathrm{A}$, should therefore also correspond to the $3 \mathrm{O}$ mode of pulsation. Note that, for simplicity, we consider here only radial modes (angular degree $\ell=0$ ), although nonradial modes are also present in LPVs. Indeed, dipole $(\ell=1)$ and quadrupole $(\ell=2)$ modes are present within sequences $\mathrm{A}$ and $\mathrm{B}$ and possibly $\mathrm{A}^{\prime}$, as discussed by Soszynski et al. (2004), Soszynski et al. (2007) and Wood (2015). Stello et al. (2014) analysed a sample of late $\mathrm{K}$ and early M type giants on the RGB which were observed by Kepler and they found their power spectra to be dominated by dipole modes for the 2 nd and higher overtones, even for the most luminous stars in their sample. This suggests that nonradial modes may also be dominant for the 2nd and higher overtones in LPVs in the OGLE sample, which overlaps in luminosity the sample used by Stello et al. (2014). Since the dipole and quadrupole periods are longer than the radial period of the same radial order, the dominance of the dipole mode could help explain why the predicted $3 \mathrm{O}$ and $2 \mathrm{O}$ periods for sequences $\mathrm{A}^{\prime}$ and $\mathrm{A}$, respectively, in panel (b) of Fig. 1 are slightly shorter than the mean observed periods for these two sequences. We are currently making nonradial pulsation models for LMC AGB stars in order to better understand the observed nonradial modes and their contribution to the period-luminosity sequences (Montalban et al., in prep).

Moving to panel (b) of Fig. 4, we see that for the stars 


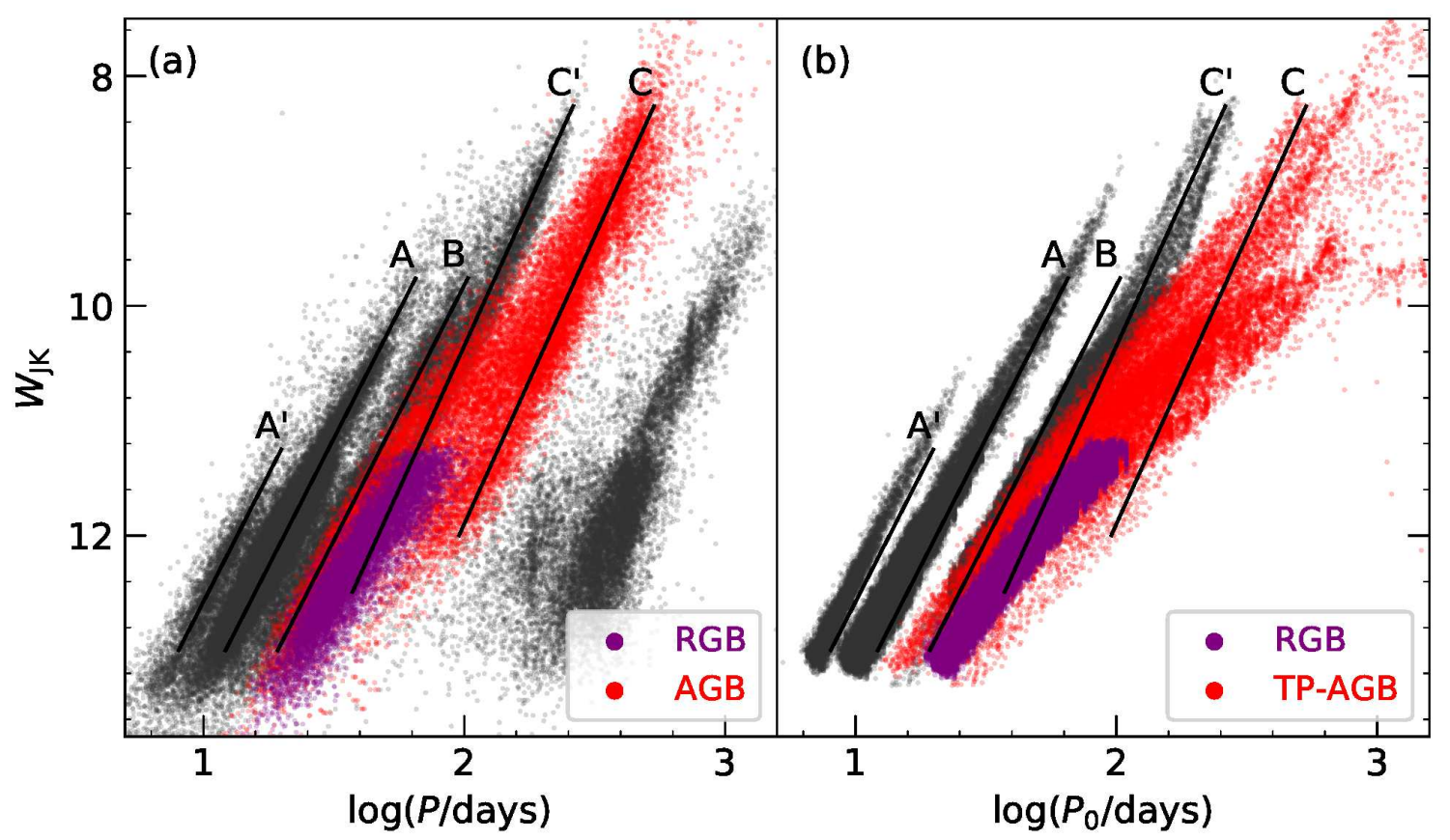

Figure 3. Panel (a). Each star in the OGLE3 Catalogue has three observed periods: primary, secondary and tertiary. Here, all of them are shown, provided that they belonging to the fourth amplitude group in Fig. 2 (labelled "fund."). They are shown as red or purple points if classified, respectively, as AGB or RGB stars (according to the OGLE evolutionary classification as in Soszynski et al. (2004)). As a reference, all primary periods are shown in the background as dark points (same as Fig. 1). Panel (b). Theoretical fundamental mode periods, regardless of their growth rates, shown in red if belonging to TP-AGB models and in purple if belonging to RGB models. Dominant overtone modes are shown as dark points in the background for reference.

whose primary period lies in sequence B, there is only one associated mode whose period is longer than the sequence B period. Once again, if this longer period mode is the fundamental mode, then the stars on sequence $B$ which exhibit the longer period correspond to 10 pulsaton. This is consistent with a picture whereby the adjacent sequences $\mathrm{A}$ and $\mathrm{B}$ are associated with adjacent radial modes. In panel (b), there appear to be three additional modes (green strips) shorter than the sequence $\mathrm{B}$ mode at low luminosities. These would be the $4 \mathrm{O}, 3 \mathrm{O}$ and $2 \mathrm{O}$ modes if all the stars on sequence $\mathrm{B}$ where $1 \mathrm{O}$ mode pulsators. However, this is not the case. In the faint part of sequence B there are in fact two groups of stars, each with a different mass at a given luminosity. The first group consists of the 10 mode pulsators mentioned above. The second group consists of more massive stars whose primary period normally belongs to sequence $\mathrm{A}^{\prime}$ and which, by chance, have their longest period mode coinciding with sequence B, as shown by Wood (2015). For these stars, the mode found on sequence B is the fundamental mode so that, at low luminosities, sequence $\mathrm{B}$ contains both $1 \mathrm{O}$ and fundamental mode pulsators. Normally, the latter group would have a primary period located on sequence $\mathrm{A}^{\prime}$ (the $3 \mathrm{O}$ mode), but sometimes the fundamental mode is detected as the primary pulsation mode, as both have rather small amplitudes.

Moving to panel $\left(\mathrm{c}^{\prime}\right)$ of Fig. 4, we see that for the stars whose primary period lies in sequence $C^{\prime}$, there is also one associated mode whose period is longer than the sequence $\mathrm{C}^{\prime}$ period, at least for AGB stars above the tip of the RGB at $W_{\mathrm{JK}} \approx 11.3$ and for some stars (possibly AGB stars) just below the RGB tip. This indicates that for these stars, sequence $\mathrm{C}^{\prime}$ corresponds to $1 \mathrm{O}$ pulsation, just like the luminous stars on sequence B. The longer period mode coincides with sequence $\mathrm{C}$ or its shorter period edge, consistent with the assumption that sequence $\mathrm{C}$ is made up of fundamental mode pulsators as argued by Wood (2015). In addition to the sequence $\mathrm{C}^{\prime}$ stars exhibiting a longer secondary or tertiary period, there are some $\mathrm{C}^{\prime}$ stars at lower luminosities that exhibit a shorter period coinciding with sequence B. These are the analogs of the sequence B stars which have their fundamental mode on or to the long period side of sequence $\mathrm{C}^{\prime}$. Thus, especially at RGB luminosities, sequence $\mathrm{C}^{\prime}$ contains some fundamental mode pulsators. As with sequence $\mathrm{B}$, the fundamental mode pulsators on sequence $\mathrm{C}^{\prime}$ are more massive at a given luminosity than the 10 mode pulsators on this sequence. Note that sequence $\mathrm{C}^{\prime}$ is sparsely populated at low luminosities and it is poorly defined there.

We now have a picture in which sequences $B$ and $\mathrm{C}^{\prime}$ are made up predominantly of 10 pulsators but at low luminosities they also contain fundamental mode pulsators of higher mass at a given luminosity. Note that this is consistent with the results of Section 2.3 based on the identification of PAL relations with the four lowest order pulsation modes. In this situation, the idea that adjacent sequences correspond to adjacent radial orders of pulsation breaks down. Another anomaly is that sequences $\mathrm{B}$ and $\mathrm{C}^{\prime}$ seem to be unusually close together in $\log (P)$. The longer period mode associated with sequence $B$ stars has a period that is longer than the period of the adjacent sequence, $\mathrm{C}^{\prime}$. For all other sequences, the secondary and tertiary periods form strips that are closer to their primary mode sequence than are the adjacent primary mode sequences. Wood (2015) argued that this is because the mass of stars at a given luminosity decreases in moving from sequence $\mathrm{A}^{\prime}$ through to $\mathrm{C}$. In this situ- 


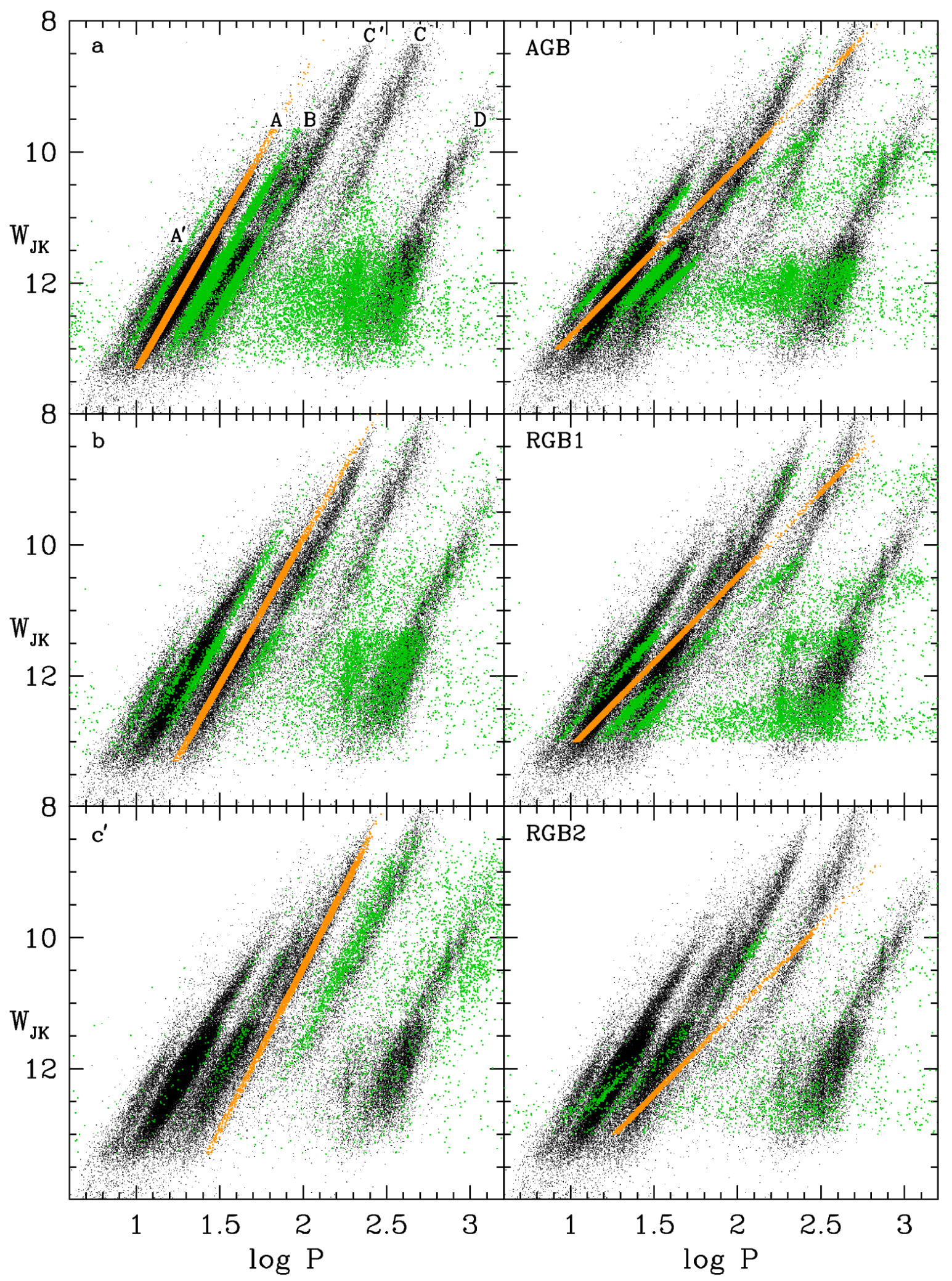

Figure 4. Plots of $W_{\mathrm{JK}}$ against $\log (P)$. Black points in each panel are the primary periods of each star. These points fall mostly on the six sequences labelled in panel (a). In the left three panels, a channel of small width centred on a sequence is defined and all primary periods in these channels are coloured orange. The secondary and tertiary periods of these stars are plotted as green points. In the right three panels, a sloping channel approximating an evolutionary track is defined and the same colour scheme is used for primary, secondary and tertiary periods.

ation, and under the assumption that sequences $\mathrm{B}$ and $\mathrm{C}^{\prime}$ corresponded to adjacent radial orders, Wood (2015) tried to explain the unusually close positions of sequences $\mathrm{B}$ and $\mathrm{C}^{\prime}$ by assuming the sequences $\mathrm{C}^{\prime}$ and $\mathrm{C}$ were ra- 
dial $\ell=0$ pulsators but that sequences $\mathrm{A}^{\prime}, \mathrm{A}$ and $\mathrm{B}$ were dominated by nonradial $\ell=1$ pulsators whose periods were slightly larger than those of the $\ell=0$ pulsators. If we relax the condition that sequences $\mathrm{B}$ and $\mathrm{C}^{\prime}$ coincide with adjacent radial orders, such a strong assumption is not necessary anymore, and we can safely assume the presence of dominant modes on sequences $\mathrm{A}^{\prime}$, A and $\mathrm{B}$ that are due to radial pulsation (although, as noted above, nonradial modes have a presence within these sequences).

In order to throw further light on the modes associated with the PL sequences, in the right hand panels of Fig. 4 we consider sloping channels in the $\log (P)-W_{\mathrm{JK}}$ diagram so that we can follow mode evolution in passing from one sequence to the next. Remember that the orange points are primary modes (the largest amplitude) and the green points are the secondary and tertiary modes (smaller amplitude).

As the orange channel crosses from sequence B to sequence $\mathrm{C}^{\prime}$ in the right hand panels of Fig. 4, we see that the fundamental mode, represented by the longest period green strip, increases in period from that of sequence $\mathrm{C}^{\prime}$ to that of sequence $\mathrm{C}$. This green strip terminates at the right hand edge of sequence $\mathrm{C}$ due to the fact that very large amplitude Mira-like pulsation occurs there and the star disappears from optical detection due to mass loss and the formation of a dense obscuring circumstellar shell. At this time, the primary mode (orange strip) lies at the long period edge of sequence $\mathrm{C}^{\prime}$. Related to this is the fact that where the orange channel (primary periods) crosses sequence $\mathrm{C}$, the secondary periods lie at the long period edge of sequence $\mathrm{C}^{\prime}$. This suggests an evolutionary sequence during which stars evolve through the regions of sequences $\mathrm{B}$ and $\mathrm{C}^{\prime}$ with the $1 \mathrm{O}$ mode as the dominant mode of pulsation and the fundamental mode as a secondary oscillation. Then at the long period edge of sequence $\mathrm{C}^{\prime}$, the fundamental mode takes over as the dominant mode and the 10 mode becomes the secondary mode. But very soon, large amplitude Mira-like pulsation occurs so that the 10 mode can no longer be detected and then mass loss occurs and the star disappears from optical detection.

\subsection{An evolutionary picture of pulsational stability}

Theoretical estimates of the stability of radial pulsation modes in red giant stars support the picture of modal evolution described above. In Fig. 5, we show the growth rate of the fundamental, $1 \mathrm{O}, 2 \mathrm{O}$ and $3 \mathrm{O}$ radial modes as a function of luminosity in a $1.6 \mathrm{M}_{\odot}$ star using the code discussed in Wood \& Olivier (2014).

At the lowest luminosities shown, all modes are unstable with lower order modes tending to have the highest growth rates and these are presumably the ones that are most likely to be the detected as the primary period of oscillation. This accounts for the prominence of sequences $\mathrm{A}^{\prime}, \mathrm{A}$ and $\mathrm{B}$ at low luminosities in Fig. 4. As the luminosity increases, the $3 \mathrm{O}$ mode becomes stable soon after its frequency exceeds the acoustic cutoff frequency $\left(\log \left(L / \mathrm{L}_{\odot}\right) \approx 3.22\right)$. This means that the primary mode of pulsation will no longer be on sequence $\mathrm{A}^{\prime}$ but will lie on sequence $\mathrm{A}$ which corresponds to the $2 \mathrm{O}$ mode which now has the highest growth rate. Similarly, the $2 \mathrm{O}$ mode becomes stable as the luminosity increases further $\left(\log \left(L / \mathrm{L}_{\odot}\right) \approx 3.55\right)$ so that the primary mode of

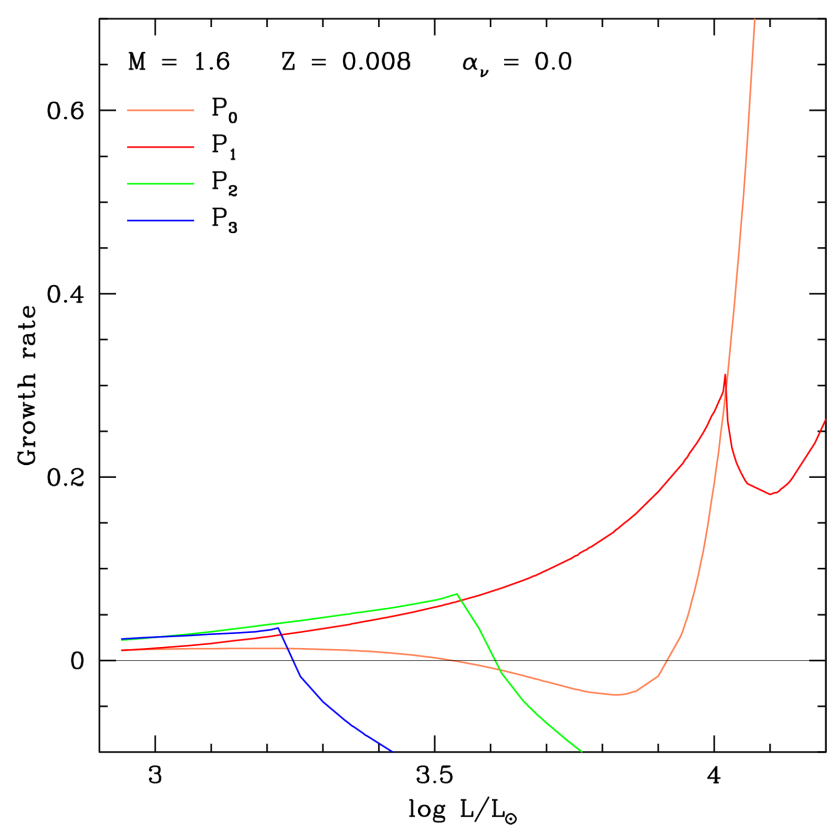

Figure 5. The growth rate of the first four radial pulsation modes in a $1.6 \mathrm{M}_{\odot}$ star as a function of luminosity. The metallicity is LMC-like $(\mathrm{Z}=0.008)$ and turbulent viscosity has been neglected. See Wood \& Olivier (2014) for details of the modelling.

pulsation now moves to sequence B which corresponds to the 10 mode which now has the highest growth rate.

The evolution of the 10 mode is somewhat different to the evolution of the $2 \mathrm{O}$ and $3 \mathrm{O}$ modes. With further increase in luminosity, the 10 mode is never stabilised by acoustic energy loss in these calculations. Note that the fundamental mode has become stable by the time the 10 mode becomes the mode with the highest growth rate. In lower mass models, the fundamental mode may not be completely stable but its growth rate is still much smaller than that of the 10 mode. At very high luminosities, the fundamental mode becomes extremely unstable. These results suggest the following picture for further evolution of the star. The star evolves so that the observed primary mode, corresponding to unstable 10 mode, moves through sequences $\mathrm{B}$ and $\mathrm{C}^{\prime}$ while at the same time the observed secondary mode, corresponding to the stable or weakly unstable fundamental mode, moves across the gap between sequences $\mathrm{C}^{\prime}$ and $\mathrm{C}$. When the star evolves to the luminosity where the fundamental mode becomes unstable, the star becomes a large amplitude Mira-like variable and the 10 mode is no longer seen (defining the long period edge of sequence $\mathrm{C}^{\prime}$ ). This is followed by mass loss and termination of giant branch evolution, thus defining the long period edge of sequence C.

\subsection{The splitting of sequences $B$ and $C$}

The problem with the picture presented so far is a lack of explanation for the distinct gap between sequences B and $\mathrm{C}^{\prime}$ even though both sequences contain $1 \mathrm{O}$ pulsators. The whole region from the short period edge of sequence $\mathrm{B}$ to the long period edge of sequence $\mathrm{C}^{\prime}$ should be populated roughly uniformly in the above picture. The solution to this problem appears to be provided by the mysterious sequence $\mathrm{D}$ variables. In the panels of Fig. 6, we show $\log (P)-W_{\text {JK }}$ plots similar to those in Fig. 4. In panels $(D-1),(D)$ and $(D+1)$, the orange channel containing 


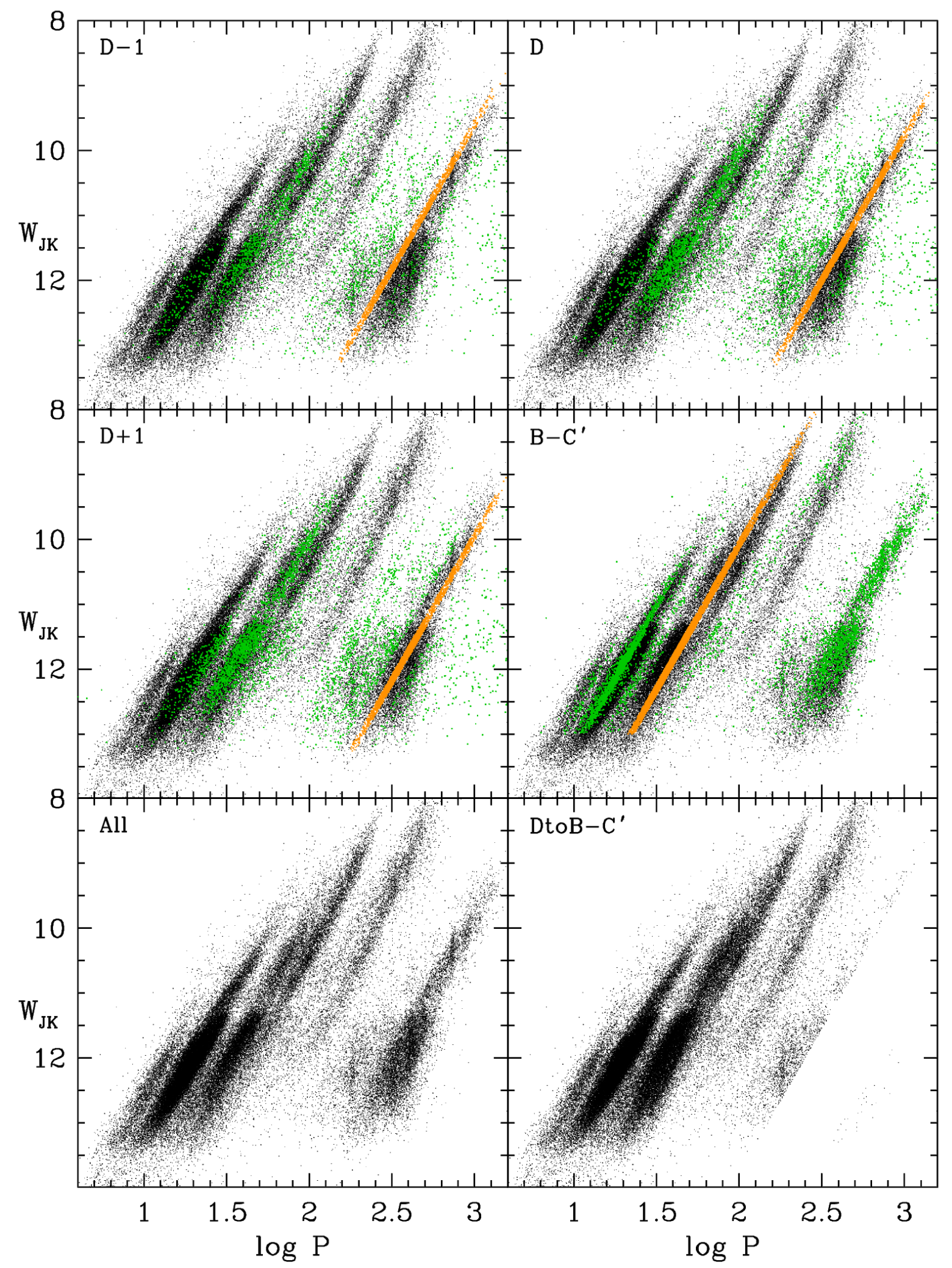

Figure 6. Similar to Fig. 4. In panels (D-1), (D) and (D+1), the channels are placed in sequence D. In panel (B- $\left.\mathrm{C}^{\prime}\right)$, the meaning of orange and green points is reversed so that the orange points correspond to secondary or tertiary periods and the green points are the primary periods of the stars marked with orange points. In panel (All), the raw OGLE III data is plotted. In panel (DtoB-C ${ }^{\prime}$ ), the sequence $\mathrm{D}$ variables have been shifted to a position lying between sequences $\mathrm{B}$ and $\mathrm{C}^{\prime}$, as described in the text.

the primary mode pulsation lies on the left, middle and right side of sequence $D$, respectively. Keeping to AGB luminosities where sequences $\mathrm{B}$ and $\mathrm{C}^{\prime}$ are both well defined, we see that the secondary modes of the sequence 


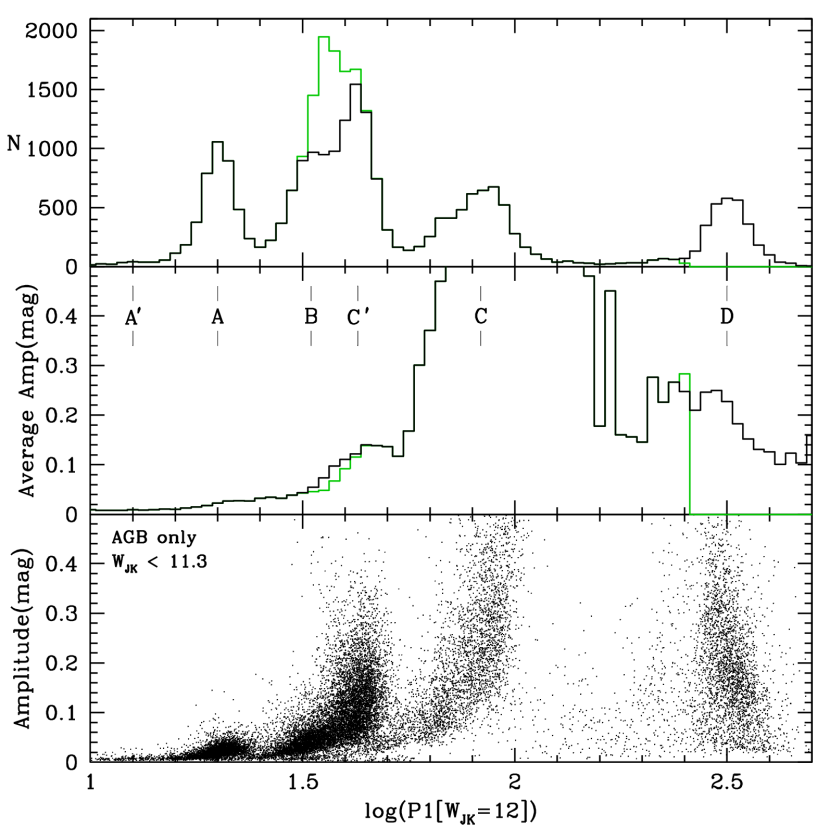

Figure 7. Bottom panel: Pulsation amplitude of individual AGB stars in the OGLE III catalogue plotted against $\log \left(P_{1, W_{\mathrm{JK}}=12}\right)$. The selected AGB stars have $W_{\text {JK }}<11.3$. Middle panel: The average amplitude of the AGB stars in bins of width 0.025 in $\log \left(P_{1, W_{\mathrm{JK}}=12}\right)$. Top panel: The numbers of stars in the bins. The positions of the PL sequences are shown in the middle panel. Black lines and points are plotted when the stars have the values of $\log \left(P_{1, W_{\mathrm{JK}}=12}\right)$ in the OGLE III catalogue. The green lines in the top panel are plotted when $\log \left(P_{1, W_{\mathrm{JK}}=12}\right)$ values for all stars in sequence $\mathrm{D}$ have been shifted to a new value between sequences $\mathrm{B}$ and $\mathrm{C}^{\prime}$, as described in the text. The green lines in the middle panel are plotted when only the $40 \%$ of sequence D stars with a secondary or tertiary period between sequences $\mathrm{B}$ and $\mathrm{C}^{\prime}$ have been shifted.

$\mathrm{D}$ variables lie between sequences $\mathrm{B}$ and $\mathrm{C}^{\prime}$ regardless of the position of the orange channel on sequence $\mathrm{D}$. In panel (B-C') of Fig. 6, we have selected secondary and tertiary periods in a channel between sequences $\mathrm{B}$ and $\mathrm{C}^{\prime}$, and the corresponding primary periods are shown as green points. It is evident that many of these primary modes coincide with sequence D variability. Therefore, there is clearly a one-to-one correspondence between sequence $\mathrm{D}$ variability and stars with oscillation periods lying between sequences $\mathrm{B}$ and $\mathrm{C}^{\prime}$. The tight correlation of the sequence $\mathrm{D}$ period with 10 pulsation period shown by the green and orange strips in Fig. 6 strongly suggests that sequence D is associated with some sort of stellar pulsation (e.g. Wood et al. 2004; Saio et al. 2015) and is not caused by a phenomenon such as binarity (e.g. (Wood et al. 2004); (Soszyński 2007)).

How the sequence D oscillations are related to 10 pulsation is not clear. However, it does seem that sequence $\mathrm{D}$ variability is the sole cause of the gap between sequences B and $\mathrm{C}^{\prime}$. Stars that would, in the absence of sequence $\mathrm{D}$ variability, be positioned in the gap between sequences $\mathrm{B}$ and $\mathrm{C}^{\prime}$, are now positioned in sequence $\mathrm{D}$. In the following, we will show that by transferring all the sequence D stars into the gap between sequences B and $\mathrm{C}^{\prime}$, the gap disappears.

To begin this process, we identify all stars having a primary period on sequence $\mathrm{D}$ and a secondary or tertiary period lying in a strip of width 0.12 in $\log (P)$ located between sequences $\mathrm{B}$ and $\mathrm{C}^{\prime}$. These stars, $40 \%$ of all stars on sequence D, are then shifted from the position of their primary period on sequence $\mathrm{D}$ to the position of their secondary or tertiary period between sequences $\mathrm{B}$ and $\mathrm{C}^{\prime}$. The remaining stars in the sequence $\mathrm{D}$ region $(60 \%$ of the original sequence D population) are also shifted into the strip between sequences $\mathrm{B}$ and $\mathrm{C}^{\prime}$ with an offset in $\log (P)$ from the centre of the strip equal to half the offset of the sequence D period from the centre of sequence D. Now all the original sequence D stars lie in between sequences $\mathrm{B}$ and $\mathrm{C}^{\prime}$. The PL sequences after this shift has been completed are shown in panel (DtoB-C') of Fig. 6. These can be compared to the original PL sequences shown in panel (All) of Fig. 6.

It is clear that after the shifting procedure, the gap between sequences $\mathrm{B}$ and $\mathrm{C}^{\prime}$ essentially disappears in the PL diagram. This can be seen more clearly by looking at the cumulative numbers of stars in narrow strips parallel to the sequences. In Fig. 7, the numbers of AGB stars (defined as those with $W_{\mathrm{JK}}<11.3$ ) in narrow strips of width 0.025 in $\log (P)$ are plotted against $\log \left(P_{W_{\mathrm{JK}}=12}\right)$. Before the sequence D stars are moved, there is a dip in the number of stars in the strips lying between sequences $\mathrm{B}$ and $\mathrm{C}^{\prime}$ (see the black lines in the top panel of Fig. 7). Furthermore, the pulsation amplitude of stars in this region increases smoothly with $P$ (middle panel of Fig. 7) suggesting that a single pulsation mode is involved. After moving the sequence $\mathrm{D}$ stars, the whole region on and between sequences $\mathrm{B}$ and $\mathrm{C}^{\prime}$ is populated as if it is a single sequence (green lines in the top panel), once again suggesting that a single mode is involved. Interestingly, the average amplitude of stars between sequences $\mathrm{B}$ and $\mathrm{C}^{\prime}$ is reduced by adding in the $40 \%$ of sequence D stars that have a secondary or tertiary period there.

We conclude that the gap between sequences B and $\mathrm{C}^{\prime}$ is actually a selection effect: the stars in this gap develop a sequence $\mathrm{D}$ oscillation that is of larger amplitude than the sequence $\mathrm{B}-\mathrm{C}^{\prime}$ oscillation and hence, in plots using the observed primary period of variability, these stars appear on sequence D rather than between sequences $\mathrm{B}$ and $\mathrm{C}^{\prime}$.

We do not know why the sequence $\mathrm{D}$ oscillation occurs. It could be due to a resonance between 10 mode pulsation and the oscillation associated with sequence $\mathrm{D}$ which drains energy from the 10 mode to the LSP oscillation of sequence D, but it could equally be a totally independent mode that just happens to have a large amplitude at this stage of evolution.

\section{SUMMARY AND CONCLUSIONS}

We have modelled a synthetic stellar population representative of red giant stars currently in the LMC, and we have assigned to each star a dominant period based on the growth rate of radial pulsation modes. We find good agreement between the theoretical PL sequences formed by the third and second overtone modes when they are dominant and the observed sequences $\mathrm{A}^{\prime}$ and A, respectively. However, the theoretical first overtone sequence spreads across both observed sequences B and $\mathrm{C}^{\prime}$, suggesting that these two sequences may in fact coincide with the same radial pulsation mode. The theoretical fundamental mode sequence obtained with this modelling agrees with sequence $\mathrm{C}$ at RGB luminosities but at AGB luminosities it has periods that are longer than those of sequence $\mathrm{C}$ which contains the large am- 
plitude Mira variables and which almost certainly does correspond to radial fundamental mode pulsation (Wood 2015)

The analysis of observed amplitudes reveals the presence of four distinct period-luminosity-amplitude relations, that we identify with the four lowest radial orders of pulsation. The amplitude distributions compare well with the distributions of theoretical growth rates of overtone modes, validating the use of the latter as proxies for amplitudes. However, as with the the PL sequences, the fundamental mode amplitude distribution shows a poor agreement with observations, especially at bright magnitudes.

If we examine where the theoretical fundamental periods fall regardless of growth rates, we find that they cover the region from the lower part of sequence B to the entire sequence $\mathrm{C}$. This is exactly the region covered by observed stars having modes associated with the observed PAL relation that we identify as fundamental mode pulsation. This suggests that while the the growth rates of the fundamental mode in the models may be unreliable and underestimated at high luminosities, the periods are generally correct.

Using an empirical examination of observations of multimode LPVs in the LMC, we have found the following. Sequence $\mathrm{C}$ consists of radial fundamental mode pulsators which are near the tip of the giant branch where the fundamental mode has become unstable. Sequences $\mathrm{B}$ and $\mathrm{C}^{\prime}$ consist of first overtone mode radial pulsators, with some fundamental mode pulsators on the lower luminosity end of both sequences. These fundamental mode pulsators are more massive than the first overtone mode pulsators on the same sequence at the same luminosity.

The gap between sequences $B$ and $C^{\prime}$ is caused by the development of a large amplitude oscillation associated with sequence D although the nature of this oscillation is unknown. Sequence A consists of second overtone mode radial pulsators and sequence $\mathrm{A}^{\prime}$ consists of third overtone mode radial pulsators. Some nonradial modes with angular degree $\ell=1$ and $\ell=2$ have also been detected on sequences $\mathrm{A}$ and $\mathrm{B}$, and possibly $\mathrm{A}^{\prime}$.

Our two approaches produce quite consistent results regarding the assignment of modes to the observed PL sequences. However, since the pulsation-population synthesis models do not include the mysterious sequence D variability, they are not able to split the first overtone pulsation sequence into the two observed sequences.

Allowing both sequence $\mathrm{B}$ and sequence $\mathrm{C}^{\prime}$ to corre- spond to first overtone pulsation means that the studies of Mosser et al. (2013) and Wood (2015) can be brought into alignment: Mosser et al. (2013) can now assign the fundamental mode to sequence $\mathrm{C}$ while Wood (2015) can now move the mode assigned to sequences $\mathrm{A}^{\prime}, \mathrm{A}$ and $\mathrm{B}$ down by one radial order to give the same mode assignment as Mosser et al. (2013). The major remaining problem with the study of LPVs is the lack of an understanding of sequence $\mathrm{D}$ variability.

We acknowledge the support from the ERC Consolidator Grant funding scheme (project STARKEY, G.A. n. 615604). This publication makes use of data products from the Two Micron All Sky Survey, which is a joint project of the University of Massachusetts and the Infrared Processing and Analysis Center/California Institute of Technology, funded by the National Aeronautics and Space Administration and the National Science Foundation.

\section{REFERENCES}

Bressan, A., Marigo, P., Girardi, L., Salasnich, B., Dal Cero, C., Rubele, S., \& Nanni, A. 2012, MNRAS, 427, 127

Girardi, L., Groenewegen, M. A. T., Hatziminaoglou, E., \& da Costa, L. 2005, A\&A, 436, 895

Ita, Y., et al. 2002, MNRAS, 337, L31

—. 2004, MNRAS, 347, 720

Kiss, L. L., \& Bedding, T. R. 2003, MNRAS, 343, L79

Marigo, P., \& Aringer, B. 2009, A\&A, 508, 1539

Marigo, P., Bressan, A., Nanni, A., Girardi, L., \& Pumo, M. L. 2013, MNRAS, 434, 488

Marigo, P., et al. 2017, ApJ, 835, 77

Mosser, B., et al. 2013, A\&A, 559, A137

Pagel, B. E. J., \& Tautvaišienè, G. 1999, Ap\&SS, 265, 461

Saio, H., Wood, P. R., Takayama, M., \& Ita, Y. 2015, MNRAS, 452,3863

Skrutskie, M. F., et al. 2006, AJ, 131, 1163

Soszyński, I. 2007, ApJ, 660, 1486

Soszynski, I., Udalski, A., Kubiak, M., Szymanski, M., Pietrzynski, G., Zebrun, K., Szewczyk, O., \& Wyrzykowski, L. 2004, Acta Astron., 54, 129

Soszynski, I., et al. 2007, Acta Astron., 57, 201

Soszyński, I., et al. 2009, Acta Astron., 59, 239

Stello, D., et al. 2014, ApJ, 788, L10

Wood, P. R. 2000, PASA, 17, 18

-. 2015, MNRAS, 448, 3829

Wood, P. R., \& Olivier, E. A. 2014, MNRAS, 440, 2576

Wood, P. R., Olivier, E. A., \& Kawaler, S. D. 2004, ApJ, 604, 800

Wood, P. R., et al. 1999, in IAU Symposium, Vol. 191,

Asymptotic Giant Branch Stars, ed. T. Le Bertre, A. Lebre, \& C. Waelkens, 151 